Eine politische Kultur (in) der Krise?

Die „letzte Generation“ der römischen Republik

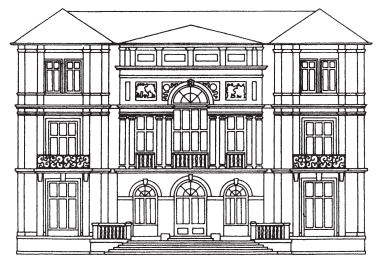




\section{Schriften des Historischen Kollegs}

Herausgegeben von Lothar Gall

Kolloquien

73

R. Oldenbourg Verlag München 2009 


\title{
Eine politische Kultur (in) der Krise?
}

Die „letzte Generation“ der römischen Republik

\author{
Herausgegeben von \\ Karl-Joachim Hölkeskamp \\ unter Mitarbeit von \\ Elisabeth Müller-Luckner
}

R. Oldenbourg Verlag München 2009 


\author{
Schriften des Historischen Kollegs \\ herausgegeben von \\ Lothar Gall \\ in Verbindung mit
}

Johannes Fried, Hans-Werner Hahn, Manfred Hildermeier,

Martin Jehne, Claudia Märtl, Helmut Neuhaus, Friedrich Wilhelm Rothenpieler, Luise Schorn-Schütte, Dietmar Willoweit und Andreas Wirsching

Das Historische Kolleg fördert im Bereich der historisch orientierten Wissenschaften Gelehrte, die sich durch herausragende Leistungen in Forschung und Lehre ausgewiesen haben. Es vergibt zu diesem Zweck jährlich bis zu drei Forschungsstipendien und zwei Förderstipendien sowie alle drei Jahre den „Preis des Historischen Kollegs“.

Die Forschungsstipendien, deren Verleihung zugleich eine Auszeichnung für die bisherigen Leistungen darstellt, sollen den berufenen Wissenschaftlern während eines Kollegjahres die Möglichkeit bieten, frei von anderen Verpflichtungen eine größere Arbeit abzuschließen. Professor Dr. KarlJoachim Hölkeskamp (Köln) war - zusammen mit Dr. Claire Gantet (Paris), Prof. Dr. Tilman Nagel (Göttingen) und Prof. Dr. Karl Schlögel (Frankfurt an der Oder) - Stipendiat des Historischen Kollegs im Kollegjahr 2005/2006. Den Obliegenheiten der Stipendiaten gemäß hat Karl-Joachim Hölkeskamp aus seinem Arbeitsbereich ein Kolloquium zum Thema „Eine politische Kultur (in) der Krise? Die ,letzte' Generation der römischen Republik“ vom 21. bis 23.Juni 2006 im Historischen Kolleg gehalten. Die Ergebnisse des Kolloquiums werden in diesem Band veröffentlicht.

Das Historische Kolleg wird seit dem Kollegjahr 2000/2001 - im Sinne einer „public private partnership" - in seiner Grundausstattung vom Freistaat Bayern finanziert, seine Stipendien werden gegenwärtig aus Mitteln der Fritz Thyssen Stiftung, der Gerda Henkel Stiftung und des Stifterverbandes für die Deutsche Wissenschaft dotiert. Träger des Historischen Kollegs, das vom Stiftungsfonds Deutsche Bank und vom Stifterverband errichtet und zunächst allein finanziert wurde, ist die „Stiftung zur Förderung der Historischen Kommission bei der Bayerischen Akademie der Wissenschaften und des Historischen Kollegs“.

historischeskolleg.de

Kaulbachstraße 15, D-80539 München

Tel.: +49 (0)892866 $3860 \quad$ Fax: +49 (0)89 28663863

E-Mail: elisabeth.mueller-luckner@historischeskolleg.de

Bibliografische Information der Deutschen Nationalbibliothek

Die Deutsche Nationalbibliothek verzeichnet diese Publikation in der Deutschen

Nationalbibliografie; detaillierte bibliografische Daten sind im Internet

über <http://dnb.d-nb.de> abrufbar.

(C) 2009 Oldenbourg Wissenschaftsverlag GmbH, München

Rosenheimer Straße 145, D-81671 München

Internet: oldenbourg.de

Das Werk einschließlich aller Abbildungen ist urheberrechtlich geschützt. Jede Verwertung außerhalb der Grenzen des Urheberrechtsgesetzes ist ohne Zustimmung des Verlages unzulässig und strafbar. Dies gilt insbesondere für Vervielfältigungen, Übersetzungen, Mikroverfilmungen und die Einspeicherung und Bearbeitung in elektronischen Systemen.

Gedruckt auf säurefreiem, alterungsbeständigem Papier (chlorfrei gebleicht)

Satz: Typodata GmbH, München

Druck: Memminger MedienCentrum, Memmingen

Bindung: Buchbinderei Klotz, Jettingen-Scheppach

ISBN 978-3-486-59053-1

Umschlagbild:

Silber-Denarius, 82 v. Chr., mobile Feldmünzstätte

(Classical Numismatic Group, Auktion vom 13.9.2006, Los 785) 